\title{
Solar flares dynamics investigation using UV diagnostics
}

\author{
I. M. Podgorny ${ }^{1}$, A. I. Podgorny ${ }^{2}$ \\ ${ }^{1}$ Institute of Astronomy RAS, Moscow, Russia \\ ${ }^{2}$ Lebedev Physical Institute RAS, Moscow, Russia
}

E mail (podgorny@lebedev.ru).

Accepted : 20 February 2018

\begin{abstract}
The photos of the pre-flare state development obtained in the spectral lines of the highly ionized iron (SDO AIA apparatus) indicate accumulation of the energy for a flare in the corona in the pre-flare local (about $10^{10} \mathrm{~cm}$ ) high temperature structures. The flare and pre-flare structures are investigated in the spectral lines of ions FeXXIV, FeXXIII, FeXVIII. A pre-flare structure appears several hours before the big solar flares. These pre-flare structures can be used for prediction of solar cosmic rays. The phenomena are well described by the electrodynamic model of a solar flare, developed on the basis of observational data and numerical magnetohydrodynamic simulation using the initial and boundary conditions, taken from active regions observation before the flare. The numerical simulations demonstrate the magnetic energy accumulation for a flare due to the current sheet creation. The spectral lines emissions demonstrate local heating of the corona during a flare up to the temperature $6.3 \mathrm{MK}, 16 \mathrm{MK}$, and $20 \mathrm{MK}$. The solar cosmic rays are accelerated in a flare. Now all proposed mechanism of cosmic ray generation are based on unproven assumptions. These assumptions are not confirmed by long-term observations. With the modern concept of cosmic rays, the fundamentally important question arises: can the mechanism of proton acceleration in solar flares explain the particles acceleration of galactic cosmic rays.
\end{abstract}

(c) 2018 BBSCS RN SWS. All rights reserved

Keywords: sun, solar flare, current sheet, spectral lines, pre-flare emission

\section{Introduction}

One of the most interesting properties of solar activity is the solar flare. The energy pulse about $10^{32} \mathrm{erg}$ can be realized. The numerical MHD simulations (Bilenko et al., 2002; Podgorny and Podgorny, 2012a; 2012b; 2013a; Jiang et al., 2016a; $2016 \mathrm{~b})$, carried out for initial and boundary conditions of the several observed flares, show current sheet creation in the corona before a flare. The current sheet is situated above an active region. During current sheet creation the magnetic energy accumulation takes place. This current sheet is created at a magnetic singular line vicinity due to magnetic disturbance arriving from the photosphere before a flare. The several magnetic structures (including magnetic rope) are considered in the plasma theory as a possible mechanism for flare energy accumulation, but numerical simulations demonstrate only a current sheet appearing. The flare can appear at this current sheet decay. The results of numerical simulations and flare observations permit to create the solar flare model (Podgorny and Podgorny, 1992; 2006; 2013b), which explains flare development. Some of big flares are accompaniment by proton events and coronal mass ejections. A similar mechanism of proton accelerate has been observed in the laboratory studies of a powerful pulsed gas discharge (Artsimovich, 1958). Protons with the energy of $\sim 20$ $\mathrm{GeV}$ accelerated in a solar flare are demonstrated with the worldwide network of neutron monitors (Balabin et al., 2005; Podgorny at al., 2010a; 2010b). This result gives us hope to get new information about the mechanism of galactic cosmic rays generation. The century-long study of cosmic ray protons and heavy nuclei that come to the Earth from the depths of the universe is not lead to understanding the physics of recorded high energy particle acceleration. The most popular acceleration mechanisms considered in many publications are associated with a shock wave. However, the possible mechanisms for cosmic rays acceleration in shock waves remain only hypotheses. The numerous alleged mechanisms of proton acceleration in the galaxy are based on unverified assumptions.

The possibility of the same mechanism for acceleration of the galactic and solar cosmic rays cannot be ruled out. This circumstance makes research of solar flares especially urgent. A number of phenomena related to acceleration of protons on the Sun and their propagation in the interplanetary space are available for observation by the modern methods. In the work of Podgorny and Podgorny, 2015; 2016 the analysis of GOES measurements of protons with the energy $\sim 100 \mathrm{MeV}$ has been carried out. The connections of registered solar proton events with the specific very strong series of flares are demonstrated in Figure 1. The flare appears above an active region after the magnetic flux of the active region becomes bigger than $10^{22}$ Max (Podgorny and Podgorny, 2011; Podgorny at al., 2013). It has been shown (Podgorny at al., 2015) that the magnetic field distribution in an active region remains practically unchanged during a flare. This means that magnetic energy dissipation during the flare occurs in the corona. It is also proven that the protons of solar cosmic rays are accelerated in the electric field appeared due to magnetic reconnection in a flare current sheet. The characteristics of the pulses of accelerated protons that recorded at the Earth's orbit strongly depend on the interplanetary magnetic field (Podgorny and Podgorny, 2015). The protons accelerated in the western solar flares arrive to the Earth along the Archimedes magnetic field lines without collision, but protons from the eastern flares arrive across the interplanetary magnetic lines with the delay of several hours. Apparently the protons propagate from the eastern flares due to collisionless diffusion at scattering 
with field fluctuation (Istomin, 2010). Figure 1 shows that not all flares are accompaniment by proton acceleration. There are no signs of a flare that can distinguish the phenomena accompanied by proton acceleration.

The typical size of the active region is about $10^{10} \mathrm{~cm}$. Active regions and individual sunspots appear on the solar disk with the eleven-year period. The number of sunspots on a visible solar disk is usually considered as a measure of solar activity. At the minimum of solar activity no active regions can be observed, but at the solar activity maximum the number of active regions on the solar disk reaches about 10. The solar activity is associated with 11 years periodic change of the solar dipole magnetic field. The solar dipole magnetic field of about $1 \mathrm{G}$ changes its polarity in the each cycle. Global cooling is observed on the Earth in the time interval with very low solar activity. The main magnetic phenomena in the Earth's magnetosphere are associated with the solar magnetic activity and clearly connect with a solar cycle. Currently, the solar activity is close to the minimum, and we use the Solar Dynamics Observatory Atmospheric Imaging Assembly (SDO AIA) data obtained in previous years.
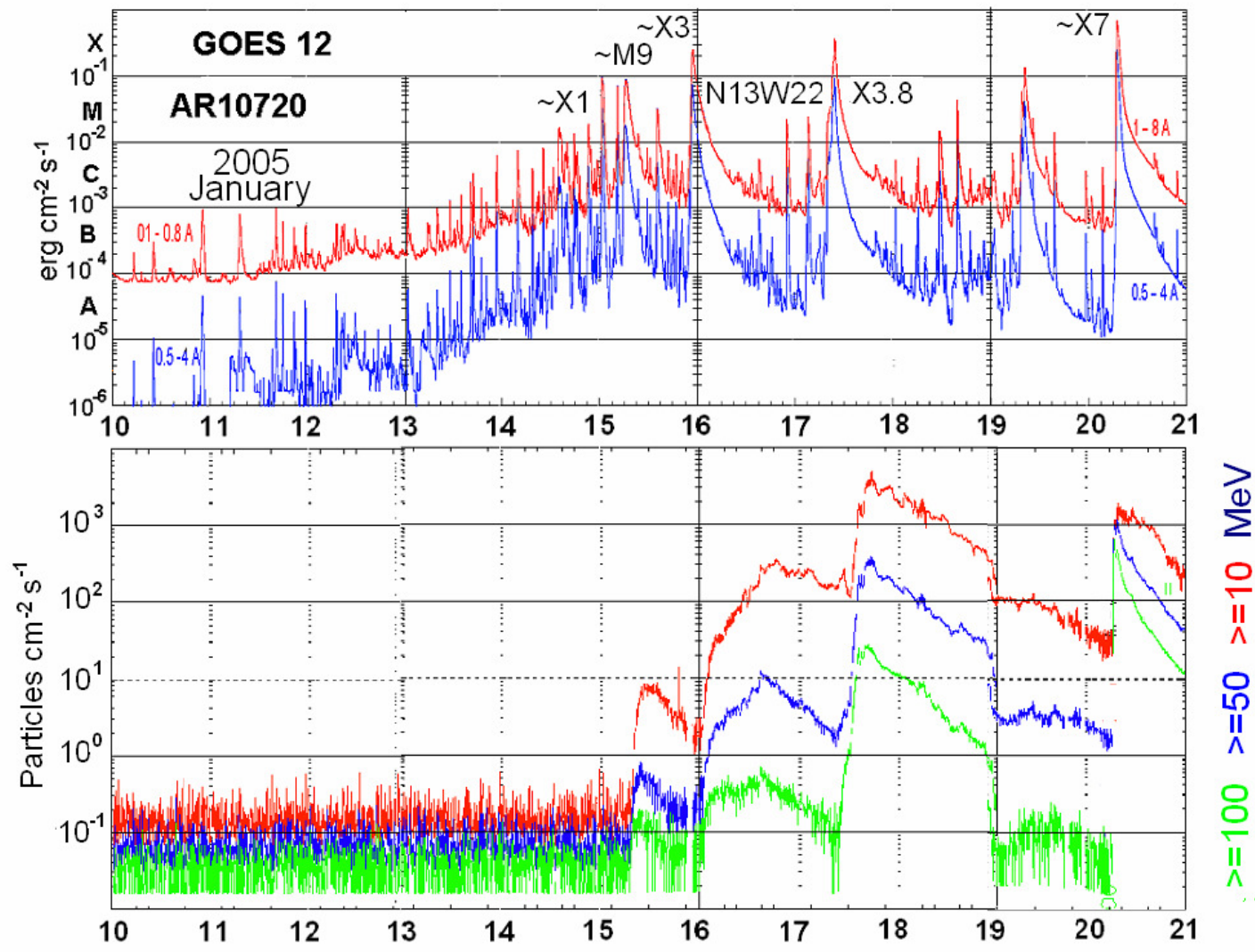

Figure 1. The strong series of solar flares. Some of flares are accompanied by a beam of relativistic protons.

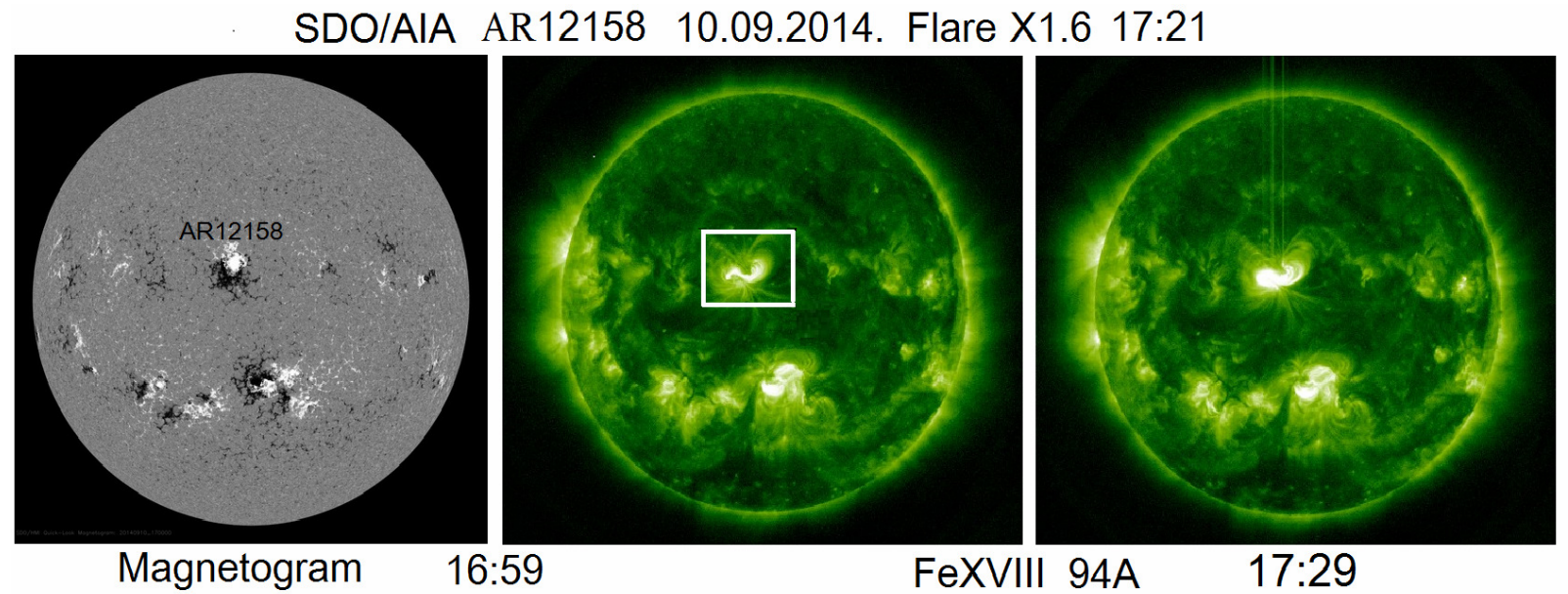

Figure 2. The solar disk magnetogram, pre-flare and flare UV emissions in the $94 \AA$ spectral line. The UV pre-flare structure is marked by the white box. 
In this paper we analyze the ultraviolet (UV) the photographs of pre-flare states and flares made by SDO AIA in the emission of the spectral lines of highionized iron ions, which allow us to judge about the place of energy accumulation before the flare and energy release during the flare. Using SDO AlA data Savicheva et al., 2012a, Saviheva et al., 2012b and Zhao et al., 2016 have been showed that the image of pre-flare states in photographs taken in the spectral lines of the highly ionized iron coincide with the quasiseparatrice calculated under various assumptions for the force-free magnetic field. There is a good agreement for AR 12158 at two hours before the flare X 1.6, but this good agreement becomes progressively worse during the event development. These works consider the possibility of magnetic field configuration appearing which coincides with the observed UV emission, but do not show existence of a pre-flare current sheet or other current systems which could produce this pre-flare emission during energy accumulation for a flare.

\section{Analysis of photographs in spectral lines of ionized iron}

According to the flare electrodynamical model (Podgorny and Podgorny, 2006), the magnetic energy accumulation in a coronal current sheet takes place in the corona above an active region. The temperature of a pre-flare structure should increase due to the current increasing in the current sheet. The pre-flare local temperature should exceed the corona temperature $\mathrm{T}>1 \mathrm{MK}$. During a flare the magnetic energy rapidly dissipates in the corona, causing strong local heating of the coronal plasma. The observational evidence for such flare development has been obtained for the first time at the X4.8 flare in 2002, observed at the eastern limb of the solar disk (Lin et al., 2003) at measurements of thermal X-ray radiation from the corona. These processes should be also observed in emission of the UV spectral lines of many times ionized iron ions, characteristic for temperature change in the solar corona. However, such data were absent then. At the present time, the photographs of the pre-flare states and the flares obtained in the UV lines of the highly ionized iron ions are contained in the SDO data archive (sdo.gsfc.nasa.gov).

The short-wave UV radiation lines $94 \AA, 131 \AA$, and $193 \AA$ are the most convenient for the flare analysis in the hot corona. The first spectral line belongs to the ions FeXVIII (the temperature 6.3 MK). The spectral line $131 \AA$ measured by the SDO spacecraft belongs to the ions FeXXIII, FeXX, and FeVIII (the temperatures $16 \mathrm{MK}$, $10 \mathrm{MK}$, and $0.4 \mathrm{MK})$. The line $193 \AA$ is produced by emission of FeXXIV and FeXII, corresponding to the temperatures $20 \mathrm{MK}$ and 1.2 MK. At the strong corona heating during a flare the particle ionization takes place, and appearing of $131 \AA$ and $193 \AA$ lines is manifested of the temperature increasing. The ions FeVIII and FeXII can not appear in the hot corona at the flare temperature increase.

The phenomena occurred in the dense chomosphere plasma, where the temperature is low $(\mathrm{T}<0.02 \mathrm{MK})$, could not produce hot plasma structures with ions FeXVIII, FeXXIII, and FeXXIV at energy accumulation in the pre-flare state. Hot spectral lines emissions demonstrate existence of the phenomena that can occur in the corona. If a solar flare is occurred in the corona at the very high temperature, then the photographs in these lines must show the fast UV emission increasing at a flare appearance above an active region.

The all solar disk photos taken 22 min before the flare $\times 1.6$ in the spectral line $94 \AA$ and the magnetogram are presented in Figure 2. The active region AR 12158 produces the solar flare X1.6. A day prior to this flare the rather bright UV pre-flare structure unlike other bright UV emissions has appeared over the AR 12158 active region in the spectral lines of the high ionized irons. The brightest emission is observed in the $94 \AA$ spectral line (T 6.3 MK). Only such bright UV structure, which is shown in the white square (Figure 2), is accompanied by a flare. The brightness of the preflare structure gradually increases during $10 \div 20$ hours. The structure is flashed at the solar flare appearing.

The dynamics of the pre-flare structures in the UV spectral lines $94 \AA, 131 \AA$, and $193 \AA$ of the highly ionized iron is demonstrated in Figure 3 . The data are obtained by processing the results emission measurements on SDO AIA and presented in the Internet (sdo.gsfc.nasa.gov). The photographs show the pre-flare dynamics above the active region and the X1.6 flare, which occurred on September 10, 2014 at 17:21 near the center of the solar disk. In Figure 3 one can see the typical hot (6.3 MK) pre-flare structure and the high temperature (20 MK) flare emissions which cannot exist in rather cold plasma of chromospheres. This bright UV pre-flare structure is situated in the corona above the active region AO12158. The hot pre-flare structure must appear in the corona, if the flare energy is accumulated in the magnetic field of a coronal current sheet, but the energy is not supplied from the Sun surface during a flare (Podgorny et al., 2013).

In all photographs in the $94 \AA$ line one can see emission increasing that appeared before the flare. The emission structure does not correspond to the shape of the magnetic lines above the active region, which are clearly seen in the photographs. The temperature of these local pre-flare emissions is much higher than the chromosphere temperature, hence the pre-flare process develops in the corona over the active region. Figure 3 demonstrates that brightest preflare emission is appeared in the spectral line $94 \AA$ ( $T$ 6.3 MK), which radiated by 18 times ionized iron ions. The emissions of the spectral lines $131 \AA$ and $193 \AA$ (16 MK and 20 MK) are not so bright before the flare. They do not show existing of the very high temperature before a flare, but they demonstrate explosive increase of the temperature emission during the flare. The typical size of observed local pre-flare structures is about $10^{10} \mathrm{~cm}$. The brightest and very fast increasing of the flare emission corresponds to the high temperature spectral line $193 \AA$, but this line is not bright at the preflare time. The fast increasing brightness of the spectral line $193 \AA$ during the flare demonstrates the fast flare energy release in the corona.

The arrow in Figure 3 below shows the time of the first frame presented, when the X-ray has been almost constant. It is not exceed $\mathrm{Cl}$. The frame shots taken before the flare clearly show the slowly increasing of 
UV emission of the heated plasma, which does not have a form of the magnetic field lines, which are seen in the photos. Only the current of a flare current system in the corona can produce such shape of the UV emission configuration, but it is not the shape of the magnetic field line with the field aligned current. The current system must heat up the plasma in the pre-flare state, when magnetic energy accumulation takes place. The emission of the pre-flare current structure is appeared at about 20 hours before the flare. MHD numerical simulations demonstrate also that a current sheet is appeared $30 \div 40$ hours before the flare. The pre-flare current sheet dynamics is accompanied by the spectral line emission of the FeXVIII over the active region. This UV spectral line emissions revealed in Figure 3 before the flare can not belong to the rather cold chromosphere. Thus, the previously conclusions about the accumulation of energy for a flare in the corona and flare production above an active region (the coronal origin of flares) are confirmed by the independent way. The flare energy release occurs due to fast magnetic reconnection, when the current sheet becomes the unstable one, and fast magnetic energy dissipation takes place. The decay of the current system should lead to fast temperature increase and explosive emission of the FeXXIV line. It is demonstrated behavior of the line $193 \AA$. This flare is accompanied by generation of solar cosmic rays. At the bottom of Figure 3 the flare development and the flow of accelerated protons are shown. The proton flow recorded on the Earth's orbit is clearly lagging in comparison with the time of the proton direct flight from the flare to the Earth, if these protons propagate along a magnetic line without collisions. In the considered event the protons from the flare on the disk center are propagating across the lines of the interplanetary space magnetic field. The delay relative to the flare is almost 10 hours.

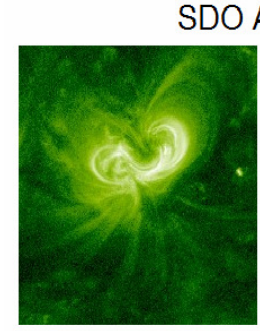

$09: 34: 32$

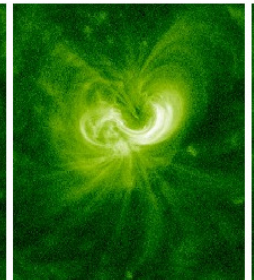

$15: 45: 02$

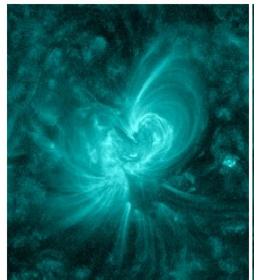

$09: 26: 34$

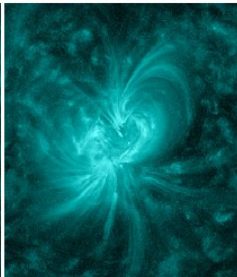

$15: 42: 46$

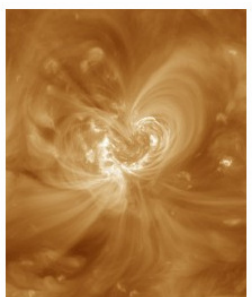

$09: 25: 19$

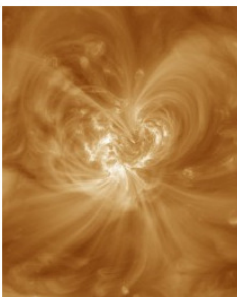

$15: 40: 19$

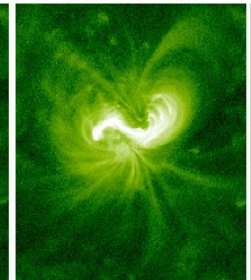

$16: 59: 38$

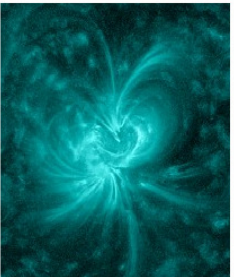

$16: 41: 58$

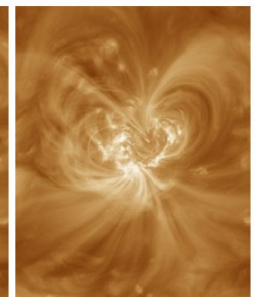

$16: 40: 43$

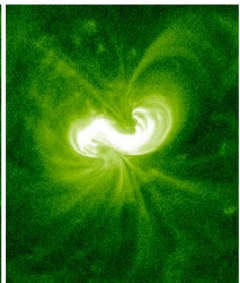

$17: 15: 02$

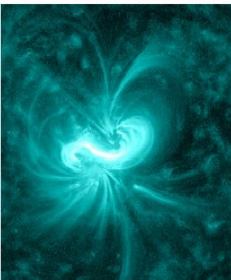

$17 \cdot 12 \cdot 46$

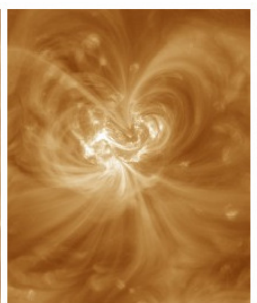

$17: 10: 55$
$94 \mathrm{~A}$ Fe XVIII (6.3 MK)

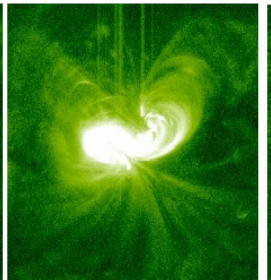

$17: 29: 14$

$17: 44: 40$

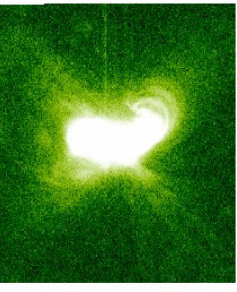

131 A FeXXIII (16 MK)

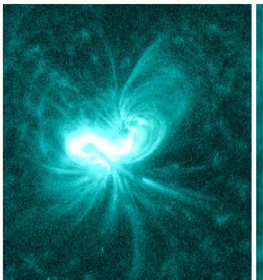

$17: 28: 12$

193 A FeXXIV (20MK)

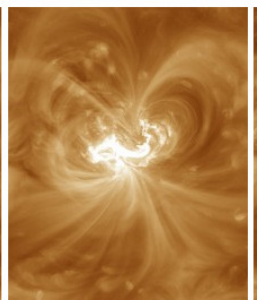

$17: 26: 42$

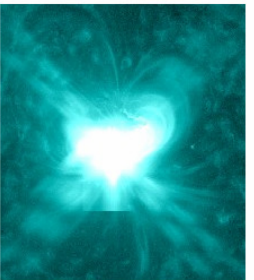

$17: 42: 46$

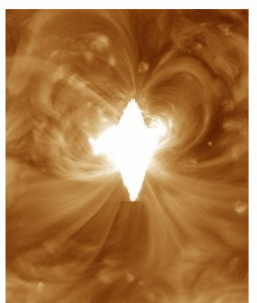

$17: 40: 43$
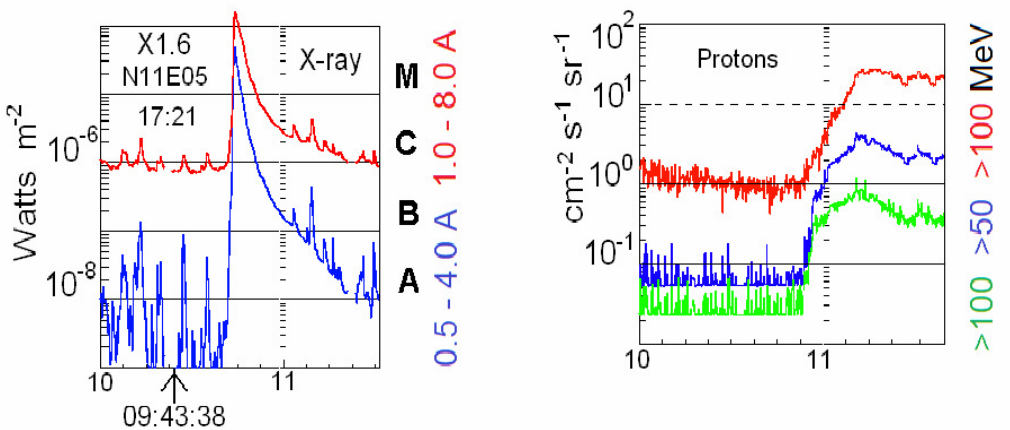

Figure 3. Photos of the active region A012158 before the flare and during the flare X1.6 from the SDO data. The emissions of three UV spectral lines of highly ionized iron are shown. The onset of the flare 17:21 is recorded by thermal X-ray radiation with GOES devices. 

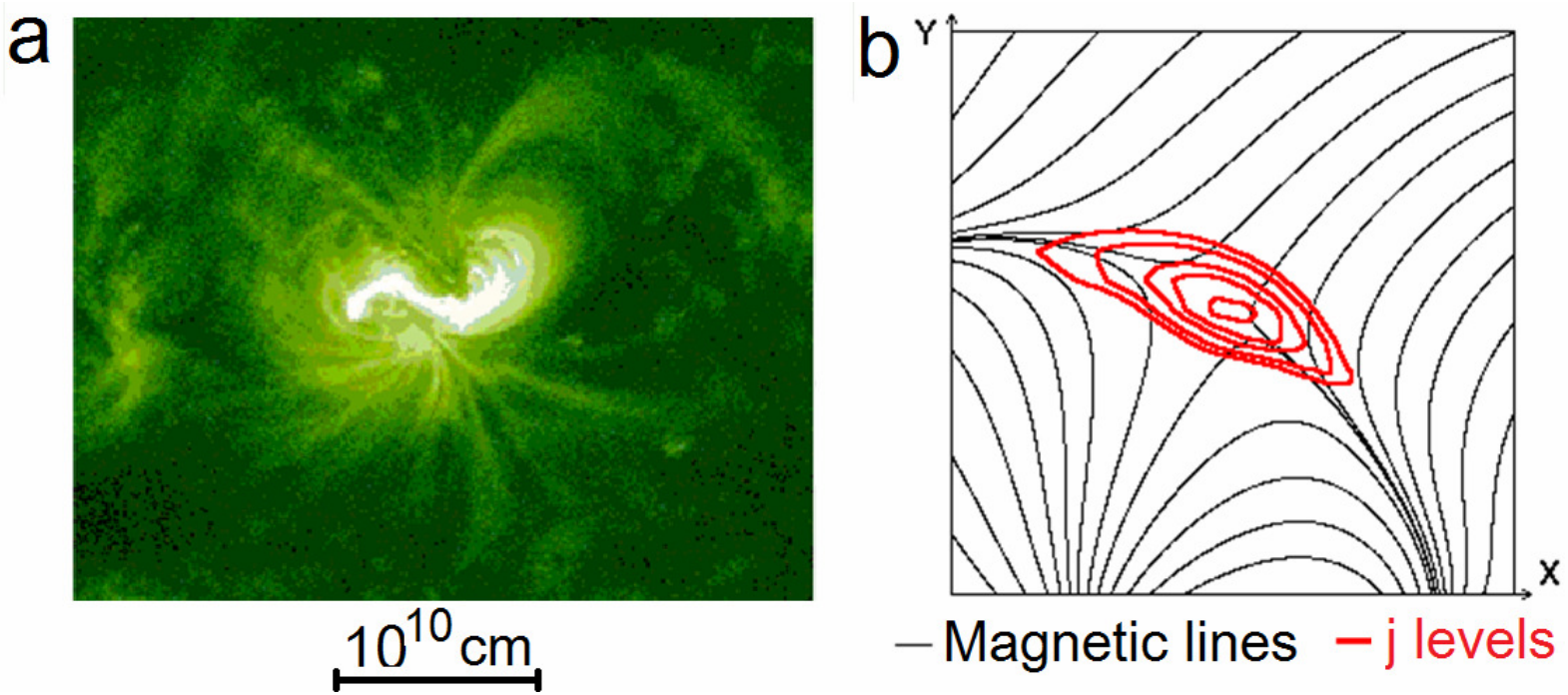

Figure 4. a). The photo of the pre-flare state in the $94 \AA$ UV spectral line. b). The pre-flare CS above AR according to numerical MHD simulation. The image of the high-temperature pre-flare structure does not correspond to a magnetic arch, but it cannot be excluded that this is a coronal current sheet.

The shape of pre-flare current structures presented in Figures 2 and 3 are observed in $\sim 50$ per cent of big flares. The structure of the image in one projection shown in the figures does not make it possible to draw an accurate conclusion about the spatial form of preflare structures. But there is every reason to assert that a hot plasma formation can not belong to the cold plasma of the chromosphere. The hot plasma must be located in the corona above an active region. We can confidently assert that the pre-flare structure is not formed along a magnetic line of potential magnetic field. The pre-flare structure has a form of the tape with length of about $10^{10} \mathrm{~cm}$. It must be located above an active region in the corona as it follows from Figure $2 \div 4$. Apparently, the pre-flare UV emission is caused at the current generation in a current sheet. The image of the pre-flare emission in an extended scale is shown in
Figure $4 \mathrm{a}$. Figure $4 \mathrm{~b}$ presents for comparison the magnetic field lines and lines of the constant current density (red lines) obtained by numerical MHD simulation at beginning of energy accumulation for a flare (Podgorny and Podgorny, 2009). The initial and boundary conditions are taken from pre-flare magnetic field measurements in the active region.

The analysis of the available SDO data shows that the pre-flare structures are appeared before all big flares, but the typical shape of the structures that can be used for flare appearance prognosis is observed only for $\sim 50$ per cent of investigated flares. The other pre-flare structures cannot be distinguished from UV structures on the solar disk that are not connected with a flare. The pre-flare structures that appeared before different flares are shown in Figure 5.

PREFLARE STRUCTURES (94 A)

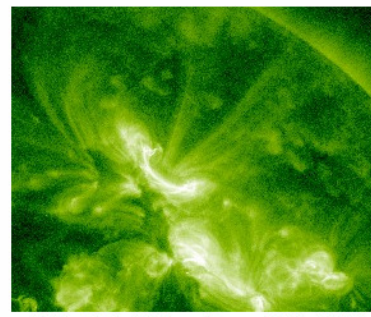

23:11:39 $\left(\mathrm{t}_{0}=01: 12\right)$

AR11420 22.01.2012 N33W21 M8.7

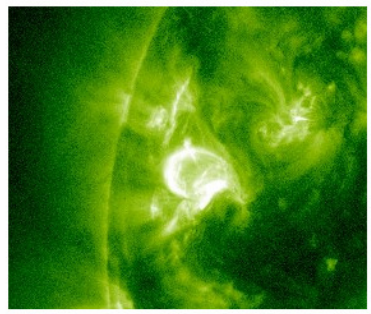

08:59:36 $\left(\mathrm{t}_{0}=09: 59\right)$

AR12059 08.05.2014 N04E42 M5.2

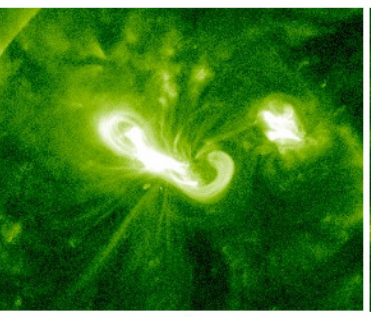

$22: 00: 03 \quad\left(t_{0}=00: 02\right)$ AR11429 07.03 .2012 N17E15 X5.4

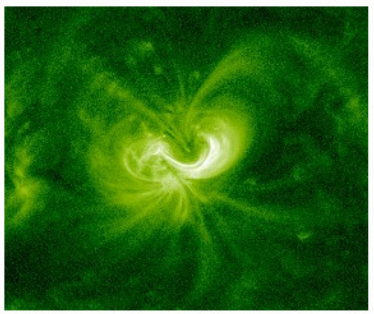

16:44:14 $\quad\left(\mathrm{t}_{0}=17: 21\right)$ AR12158 10.09.2014 N11E05 X1.6

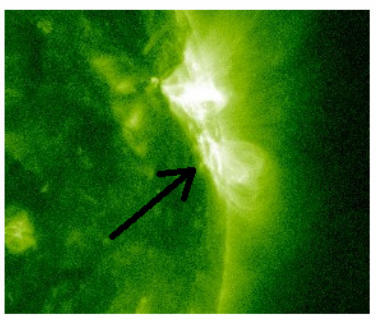

$00: 00: 15 \quad\left(t_{0}=01: 25\right)$ AR11476 17.05.2012 N12W91 M5

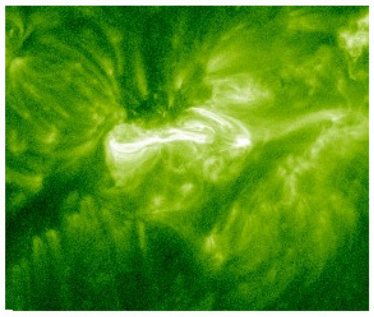

07:13:26 $\quad\left(t_{0}=09: 31\right)$ AR12242 19.12.2014 S18W42 M1.3

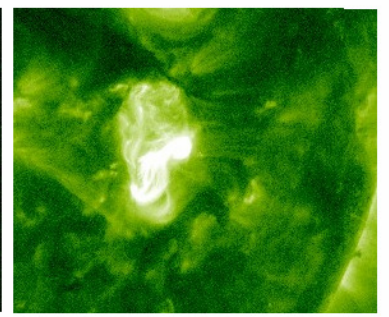

$11: 59: 50 \quad\left(t_{0}=12: 31\right)$ AR12036 18.04.2014 S15W42 M7.3

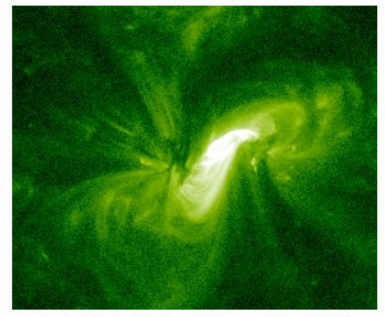

11:00:00 $\quad\left(t_{0}=11: 20\right)$ AR12473 28.12.2015

S23W11 M1.9

Figure 5. Photos obtained in the $94 \AA$ spectral line before the different flares. 
sepember, 22.2011 .

Hel 304A

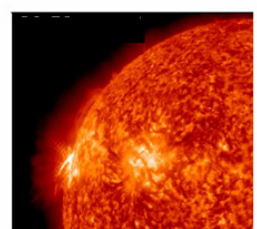

08:59:57

FeXVIII 94A

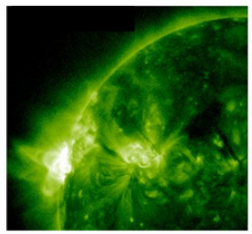

08:59:39

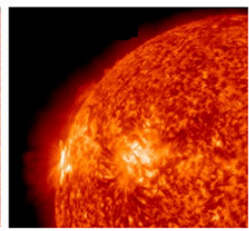

09:58:45

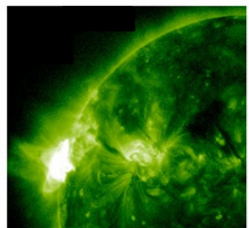

09:58:27

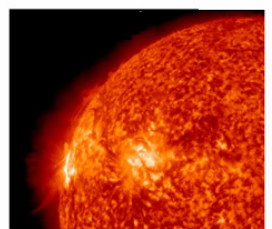

10:28:57

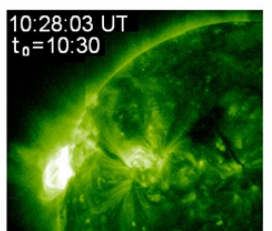

10:28:57

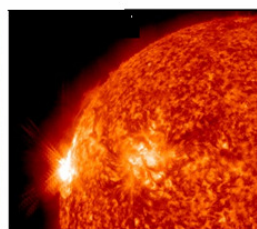

$10: 59: 57$

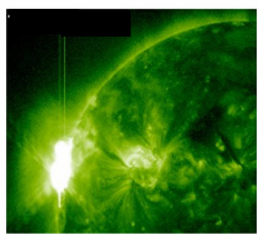

10:59:57

\section{C8.9 N15E88 $t_{0}=8: 49$}

AR11302 X1.4 N09E89 $t_{0}=10: 30$
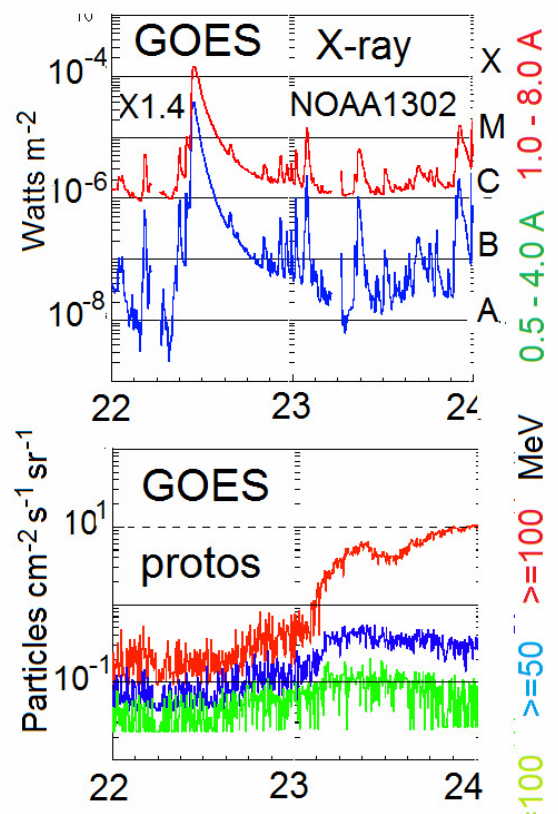

Figure 6. The pre-flare state on the eastern limb and the flare in the UV spectral lines emissions of the cold chromosphere and hot coronal plasma.

\section{The flares on the limb.}

The flare appeared on the limb of a solar disk makes it possible to observe the energy accumulation and flare energy release in the corona outside the solar disk, when influence of the solar disk radiation is completely ruled out. The flare on July 23, 2002 that is very convenient for observing in the corona has been investigated (Lin et al., 2003). It was clearly shown that the X-ray source of the thermal X-ray ( 20 MK) X4.8 flare had been located in a coronal cloud with the plasma density of $10^{11} \mathrm{~cm}^{-3}$ and the number of particles order of $\sim 10^{37}$. Despite this Lin et al. fundamental result, a number of papers have been published recently in Russia (Zaitsev et al., 2015), in which it is stated that flares do not occur in the corona, but the flare is a typical chromospheric phenomenon.

Unfortunately, there are no photographs in various UV spectral lines for the 2002 year flares showing a preflare state at different plasma temperatures. The rare flare event is shown in Figure 6, where the flare occurs in the corona outside the eastern limb, as it had been observed in (Lin et al., 2003). The flare dynamics on the eastern limb of the solar disk is demonstrated in UV photographs of the flare $\times 1.4$ which appeared September 22, 2011. These photographs are shown in Figure 6. The photos in the upper line are obtained in the spectral line of the ionized helium Hel $304 \AA$. The very weak pre-flare emission at the temperature $\sim 50$ $000 \mathrm{~K}$ can be seen on the disk. The other pre-flare behavior is demonstrated by the emission of the FeXVIII $94 \AA$ spectral line of the hot plasma, which corresponds to the temperature of $\sim 6.3 \mathrm{MK}$. The temperature 6.3 MK is considerably exceeding the usual corona temperature. The image in this line $\sim 1010$ $\mathrm{cm}$ is appeared in the pre-flare state in the corona, when according to numerical MHD simulations the magnetic energy is accumulating for a flare. During the flare, the brightness of this image is sharply increased. The flare energy release takes part in the corona beyond the boundaries of the solar disk.

The pre-flare emission of Hel $304 \AA$, which is barely noticeable on the solar disk surface and bight pre-flare emissions of the hot $94 \AA$ line of FeXVIII above the solar surface demonstrate localization of flare phenomena in the corona above an active region. These photos leave no doubt about the coronal origin of the flare. This eastern flare (Figure 6, below) is accompanied by a flux of solar cosmic rays with slow increasing front and the delay of $\sim 20 \mathrm{~h}$, because the protons could propagate to the GOES spacecraft only across the magnetic lines of the interplanetary magnetic field due to scattering with plasma inhomogeneity.

Figure 7 shows development of the $94 \AA$ and $193 \AA$ spectral lines emissions at the M5 flare appearance on the western limb. Apparently a significant portion of the active region AR11476 has been situated on the back side of the Sun during this flare. The bright preflare structure in the line $94 \AA$ is appeared in the corona only about 10 minutes before the flare start. The strong emissions in the lines $94 \AA$ and $193 \AA$ are appeared at the flare X-ray maximum ( 02:00). The hot plasma cloud creation during the flare in the solar corona outside the western boundary of the solar disk manifests itself. The positions of pre-flare emission and explosive appearing of the flare in the spectral line 193 $\AA$ are shown by the black arrows. The picture clearly indicates that the flare energy accumulation and this energy fast release take place in the solar corona. The flare is not located in the chromosphere.

The western flare is accompanied by the flow of solar cosmic rays with a sharp front, which arrive with a delay relative to the front of the flare not longer than $20 \mathrm{~min}$. This means that the flow front of protons arrives from such flares to the Earth without collision along the magnetic field lines of the Archimedes spiral. The delay of arrival to GOES $20 \mathrm{~min}$ is typical for proton flow fronts coming from the West for all investigated western flares. 


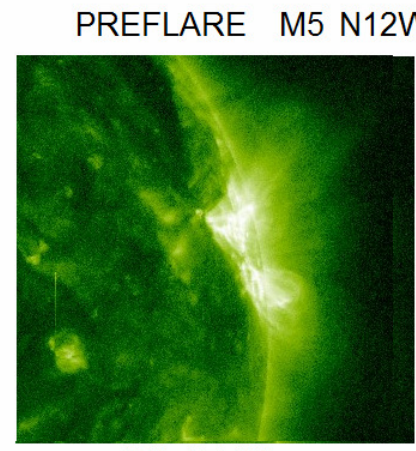

23:17:39

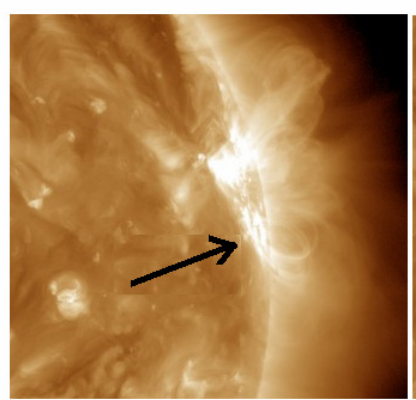

$23: 15: 32$

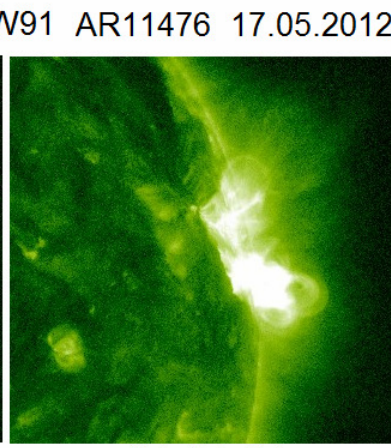

$01: 17: 15$

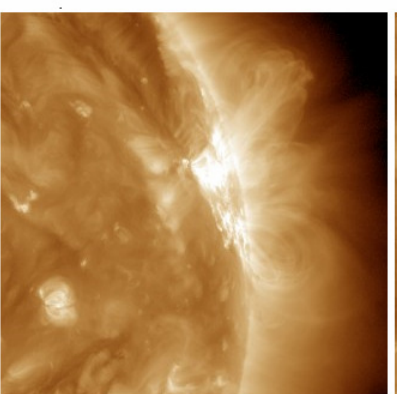

01:15:56

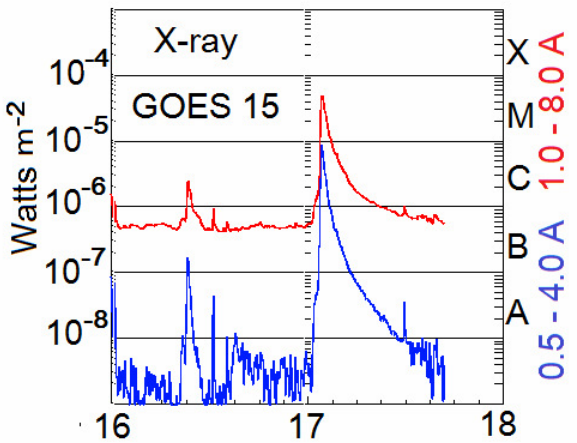

$t_{0}=01: 25$

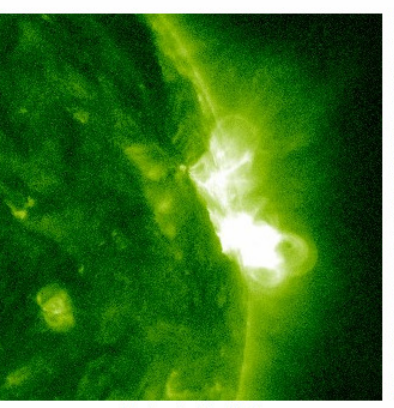

01:32:39

$94 \mathrm{~A} \quad \mathrm{FeXVIII} \quad 6.3 \mathrm{MK}$

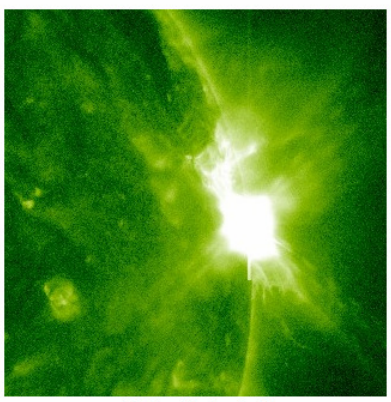

02:01:15

$193 \mathrm{~A}$ FeXXIV $20 \mathrm{MK}$

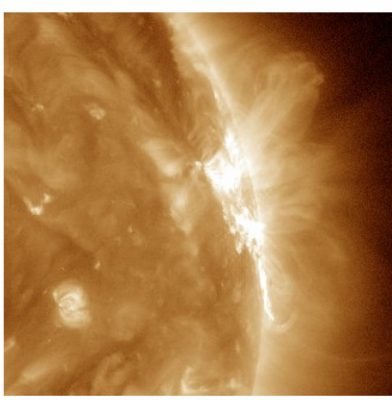

01:30:34

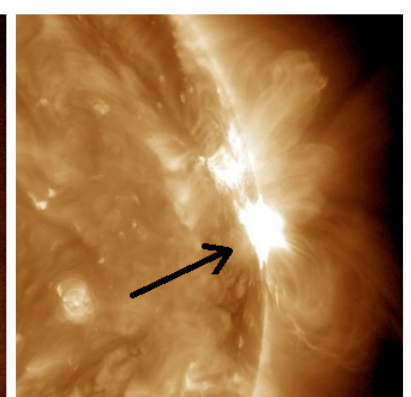

02:0020

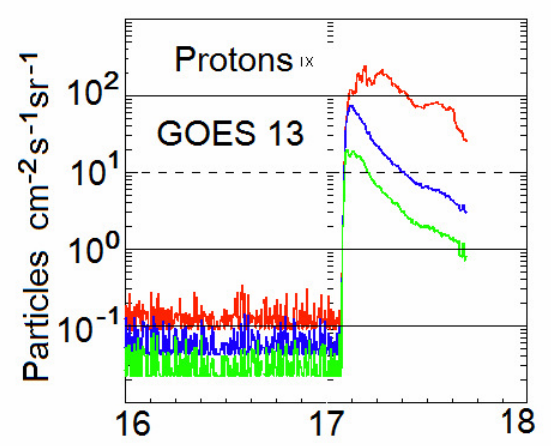

Figure 7. Pre-flare condition and the M5 flare in the coronal lines $94 \AA$ and $193 \AA$ from SDO data. The graphs show development of the flare thermal $X$-ray radiation and solar cosmic rays according to the GOES data.

\section{Conclusion}

The appearance of a local source of the UV emission of highly ionized iron ions in the corona above an active region before the flare and during the flare is demonstrated. The systematically recorded data of the SDO space craft are used. The emissions of the UV spectral lines of ions FeXVIII and FeXXIII in the corona that appear $\sim 20$ hours before a flare are observed. At that time the numerical MHD simulation shows the current sheet creation and the magnetic energy accumulation above an active region. The brightest pre-flare structure with length $\sim 10^{10} \mathrm{~cm}$ is demonstrated the spectral line $94 \AA$ (FeXVIII), corresponding to the temperature $\sim 6.3 \mathrm{MK}$. The flare appearance can be predicted by the appearance of such emission. The brightness of the plasma structure in the spectral line $193 \AA$ (FeXXIV) sharply (within 10 min) increases during a flare. The restricted plasma cloud above the active region reaches the temperature $\sim 20$ MK during the flare. At the same time, the local temperature of the chromosphere increases only slightly. The energy accumulation for a flare and flare energy release takes place in the solar corona. The sharp (several minutes) increase of the UV spectral lines emission in the corona above the Sun surface is clearly observed for flares on the solar limb. The strong plasma heating above the active region cannot be explained by the flare energy release on the surface of the Sun in the chromosphere. The flare energy release takes place in the corona.

The pulses of solar cosmic rays that created in the western and eastern flares have the characteristic shapes that typical for flare position on the solar disk. The rapid arrival of protons and the steep front of the proton flow from the western flare is determined by propagation without collisions of particles along the magnetic field lines of the Archimedes spiral. The delayed flow of protons from the eastern flare has a gently sloping front. Solar cosmic rays from the eastern flares can propagate to the Earth only across the magnetic field. 


\section{References}

Artsimovich L.A.: 1974, Controlled thermonuclear reaction. Gordon and Breach. New York.

Balabin Yu.V., Vashenyuk E.V., Mingalev O.V., et al.: 2005, Astronomy Reports. 49, 837.

Bilenko I.A., Podgorny A.I., Podgorny I.M.: 2002, Solar Phys. 207, 323.

Istomin Ya.N.: 2010, Mon. Not. R. Astron. Soc. 408, 1307.

Jiang C., Wu S.T., Feng X., Hu Q.: 2016a, Nature Communications. 7 , id. 11522.

Jiang C., Wu S.T., Yurchyshyn V., Wang H., Fen, X., Hu Q.: 2016b, Astrophys. J. 828, article id. 62, 12.

Lin R.P., Krucker S., Hurford, G.J. et al.: 2003, Astrophys. J. 595, L69.

Podgorny I.M., Balabin Yu.V., Vashenuk E.M., Podgorny A.I.: 2010a, Astron. Reports. 54, 645.

Podgorny I.M., Balabin Yu.V., Podgorny A.I., Vashenyuk E.V.: 2010b, JASTP. 72, 988.

Podgorny, A.I. and Podgorny, I.M.: 1992, Solar Phys. 139, 125.

Podgorny A.I., Podgorny, I.M.: 2006, Astronomy Reports. 50, 842.

Podgorny A.I., Podgorny, I.M.: 2009, Phys, of Auroral Phenomena. Proc. 32-nd Annual Sem. Apatity. P. 123.

Podgorny A.I., Podgorny I.M.: 2011, Astronomy Reports. 55, 629.

Podgorny A.I., Podgorny I.M.: 2012a, Geomagnetism and Aeronomy. 52, 150.

Podgorny A.I., Podgorny I.M.: 2012b, Geomagnetism and Aeronomy. 52, 162.

Podgorny A.I., Podgorny I.M.: 2013a, Sun and Geosphere. 8, 71.

Podgorny A.I., Podgorny I.M.: 2013b, Geomagnetism and Aeronomy. 53, 966.

Podgorny A.I., Podgorny I.M.: 2015, Astronomy Reports. 59, 888.

Podgorny A.I., Podgorny I.M.: 2016, Sun and Geosphere. 11, 86.

Podgorny I.M., Podgorny A.I., Meshalkina N. S.: 2013, Sun and Geosphere. 8, 63.

Podgorny I.M., Podgorny A.I., Meshalkina N. S.: 2015, Astronomy Reports. 59, 795.

Savicheva A.A., van Ballegooijen A.A, DeLuca E. E.: 2012a, Astrophysical J. 744, 78.

Savicheva A.A., Pariat E., van Ballegooijen A., at al.: 2012b, Astrophysical J. 750, 15.

Zhao J., Gilchrist S.A., Aulanier G., et al.: 2016, Astrophysical J. $823,62$.

Zaitsev V.V., Kronshtadtov P.V., Stepanov A.E. 2015. Solar and Solar-Terrestrial Physics - 2015. S-Petersburg, Pulkovo. P. 149 